\title{
Dynamic Simulation for Sustainable Transportation System
}

\author{
Hassan M. Hassoon ALDelfi
}

\begin{abstract}
This research is intended to simulate the already developed mathematical model for the five means of transportation systems which are bicycle, water transportation, taxi, bus, and metro. The resulting ecological footprint effect is being used to reflect those means of transport on the environment. A simplified approach to those means is represented by bicycle sustainable impact (BSI). In order to testify this newly derived mathematical model, the equivalent effects of different transportation systems on the environment are represented. The simulation is applied to five randomly selected cities of different geographical locations. They are considered to optimize the sustainability and hence the Anti-Sustainability collectively for the existing transportation in those cities. The selected cities are Erbil (Iraq), Baghdad (Iraq), Maysan (Iraq), Dubai (UAE) and Glasgow (UK). The five randomly selected cities have different modes of transportation. Thus different Anti-Sustainable Impact (ASI) has been found. Conclusive remarks have been drawn accordingly to achieve safe and distinguished sustainable guidelines. High-quality power run metro system depicts higher Anti-Sustainable Impact, Those guidelines can be implemented in future traffic and transportation systems evaluations in any city.
\end{abstract}

Index Terms-Sustainable transportation, dynamic simulation, BSI, mathematical modeling, transportation systems anti-sustainability.

\section{INTRODUCTION}

Transportation is a vital mean for mass transporting people and freight across our planet earth, it is in the same time considered as the source of side effects of pollutants namely particulates, NOx, COx, and SOx these pollutants are emitted when fuel is burnt in partial conditions or when the starting of engines or in the process of slow traffic jam. It can be said also that engines of low efficiency, large size and the loads that carry, are all major factors for pollutants emissions [1].

In the later years of the second part of the last century, the term sustainability has taken an important space in the development, operations of systems and other aspects of applications. That has been done, to reduce the pollution that results and to preserve environment [2]. The transportation sector in no doubt constitutes a noticeable source of pollutions and energy usage this sector represents $40 \%$ of energy consumption [3]. The sustainability depicts how to preserve bio-diversified and productive earth systems all the year round for us as human population; sustainability means the ability of mankind to preserve the environment as intergenerational equity. There are ten millions of species in

Manuscript received August 11, 2018; revised December 7, 2018.

H. H. ALDelfi is with the Faculty of Engineering, Ishik University, Erbil, Iraq (e-mail: hassan.hassoon@ishik.edu.iq). our planet earth, out of those only 1.6 million have been discovered, the human is one of them, and human is the only creature who is the main causer for environmental damage and the cause for the affluenza and environmental degradation [4]. The awareness of this deterioration has come about since the start of the industrial revolution in Europe and elsewhere, population change and overconsumption at an alarming rate. Considerable changes in the environment have been caused by the introduction of new products, machines, and new transportation systems. Despite the international protocols laid by the world general assembly and other universal bodies, still many countries are not abided by those laid protocols. If we were to take the forbiddingness of the leaded petrol in cars that first initiated by the Scandinavian countries, we can see many countries in Asia and Africa are far away from implementing this in their transportation systems. The effects of such fuels will have a negative setback on the newly born children and more. Another example that has drastic effects on environment is the still concurrent usage of the CFC (cloro-floro-carbon) refrigerants that are still being used in transportation systems and household equipment like refrigerators and so forth, yet again many countries in Asia and Africa still manufacturing and using those non-friendly environment refrigerant gases that damaging out the Ozone and the stratosphere layer.

A smooth traffic flow will certainly reduce energy waste and pollution. Highways, flyovers, road regulations, allocating lanes for an integrated public transportation and emergency services and geographical relocations of public establishments and the usage of solar powered digital systems all means towards sustainability. The type of transportation used in a given place will in no doubt influences the degree of affluence in energy usage by given population, equation (1) introduced by reference [5], This relation describes the resulting environmental impact as a function of population, affluence, and the technology in use.

$$
I=P A T
$$

where

$\mathrm{I}=$ the resulting environmental impact

$\mathrm{P}=$ population size of a given place.

$\mathrm{A}=$ the affluence

$\mathrm{T}=$ technology being in use.

Equation (1) was considered by the author as the stepping stone which led to the development of a mathematical model for the different transportation systems in this study.

The ever increasing population, the technology which is being used which is represented by different types of transportations. Those types of transportation systems have 
different carrying capacities. The various types of such transportation systems and its respective carrying capacities constitute the prominent factors in this study which lead to the respective resulting environmental impact. The above formula will clearly reflect the resulting environmental impact, If we are able to compare two families say, one family's living style is off down to earth and the other of an upper-class family residing in huge villa with many four-wheel drive cars and other household equipment employing many servants, it is in no doubt, the comparison will lead to a proportional increase of the side effect on the environment, this comparison will reflect the clear difference between sustainable and unsustainable living. However, the planet earth is considered as a closed environmental system to all matters transfer, that is to say, any environmental shortcoming in any place on this planet will surely affect any other place elsewhere in this planet. Any environmental problem will face no difficulty in crossing into other countries of the world we are living in. Environmental problems need no visa to cross, environmental problems cannot be halted by any physical boundaries, there are many examples which justify this statement: the Fukushima Daiichi nuclear disaster was an energy accident at the Fukushima Daiichi On the 5th July 2012, and the Chernobyl nuclear disaster On April 26th, 1986, both accidents overshadowed vast areas of this world. It can be said then with no minimum doubt that the environmental issues are of a universal nature thus they are international problems; mankind has to collaborate to deal with. The fossil-fueled transportation systems are those issues we have to tackle in collective and collaborative efforts.

The ever-increasing population growth where population is exceeding the seven billion, and hence the increased demands for usage of land, services and transportation, the immigration from countryside to cities, the usage of fossil fuels to boost economic growth and hence economic development and the resulting environmental pollution, the increasing demand for sources of energy and the alarming environmental degradation all together led to pursue this study. The type of transportation systems used in any given place will in no doubt influences the degree of affluenza in energy and materials consumption by any population. Reference [5] quoted a relation which depicts proportionality between the environmental impact which is linearly related to the people' living style, their degree of affluence and the technology being used, reference [6] developed their implicit model through interaction introduced transportation system in interactive links between society, environment and economy. Reference [7] introduced the Sustainable Transportation Performance Indicators (STPI) of indices for sustainable transportation; all related to the effects and limitations of the transportations systems on different aspects of energy consumption, usage, emission, and people movements. On the other hand reference [8] reported on the development of an initial set of 14 Sustainable Transportation Performance Indicators (STPI). These indicators are designed to show movement of transportation in Canada towards or away from sustainability The Dow Jones Sustainability Indices (DJSI) launched in 1999, are a family of indices evaluating the sustainability performance of the largest 2,500 companies listed on the Dow Jones Global Total Stock Market Index [9] for assessing issues such as corporate governance, risk management, branding, climate change mitigation, supply chain standards and labor practices. The trend is to reject companies that do not operate in a sustainable and ethical manner. It includes general as well as industry-specific sustainability criteria for each of the 58 sectors defined according to the Industry Classification Benchmark (ICB) [10].

Once again, all interpretations leave implicit approach to describe the highly complex domains of environmental sustainability. The author intended to simplify linearly a comparative sustainability and anti-sustainability reactions to the use of transportation systems. The proposed linear model takes into consideration, the energy quality input to any transportation system. A bicycle is considered of the upper end for sustainability and the most polluting energy consuming transporting system like the metro system at the lower end of the scale of 5 units. The derived mathematical model is simulated on five transportation systems all show their anti-sustainable environmental impact in a simplified approach. This simplified linear model is developed in this paper to simulate the usage of different transportation systems in different countries of the world; furthermore, it can be made ready for further implementations in non-linear complex environmental processes.

Once again this model as it has been suggested by the author earlier, a five systems of transportation are being considered, they are bicycle, water transport, taxi, bus, and metro. From experience and notifications of usage of such transportation systems by the author, the carrying capacity of each transportation system is specified accordingly. Further simplification is assumed to reflect the individual system on the environmental effect and impact of those transporting systems on environment through ( 1 to 5 ) scale (see Table I), this scale is introduced based on the degree of high-quality energy used in driving the transportation system. Where 5 is classified as of sustainable impact value, because of the fact low-quality energy is utilized ( the solar capital) which is the case with cycling, on the other hand, Metro system reflects usage of very high-quality energy, in this system oil energy is passed through various processes starting from the extraction, refining transportation, storage combustion, power generation then distribution to be reached to metro as high-quality energy to provide electricity to drive the metro system; thus more bad effects on the environment are experienced throughout the whole processes. The following (see Table II) depicts the rank of various quality energy used in five systems, metro, bus, taxi, water boat transportation, and bicycle respectively. It is a common practice to evaluate any product or process scaling probability from 1 to 5 , such that the worse is a metro and the best is the bicycle or bike. The Environmental Impacts for Different Transportation Systems and the Degree of Sustainability put as such: Very Bad Impact $=1$ for Metro Bad Impact $=2$ for Bus, Medium Impact $=3$ for Taxi, Acceptable Impact $=4$ for Water boat and Sustainable Impact $=5$ for Bicycle. The driving energy and the transportation impact affecting the environment and infrastructure and its availability and whether it is using high or low-quality energy are all considered in this methodology. 
Table I depicts the rank of the degrees of sustainability already proposed for the five transportation systems, metro, bus, taxi, water boat and bicycle respectively.

TABLE I: THE DEGREES OF SUSTAINABILITY FOR VARIOUS TRANSPORTATION SYSTEMS

\begin{tabular}{lcl}
\hline \hline Degree of Sustainability & Transportation System \\
\hline Very Bad Impact & 1 & Metro (50 person/wagon) \\
Bad Impact & 2 & Bus (50 persons load) \\
Medium Impact & 3 & Taxi (4 persons load) \\
Acceptable Impact & 4 & Water (20 persons load) \\
Sustainable Impact & 5 & Bicycle (one person load) \\
\hline \hline
\end{tabular}

Generally speaking, in constructing any model of any transportation system, one has to question many factors whether this transportation system is on a sustainable path. In this case, an answer for the four principles of sustainability has to be implemented, those principles are: the solar capital, the chemical recycling, the bio-diversities, and the population growth are all to be considered [11]. Another important consideration is the technology, the economical consideration and feasibility for providing the driving energy to any transporting system; those are categorized as high or low-quality energy and high or low-quality matter. Both types give different concepts in its availability and impact on the environment, society and of course on the economy, consequently, all will lead to a negative impact on the environment and sustainability. The author looked into more practical prospective by categorizing the environmental impacts by the different transportation systems in a scale of classes ( 1 to 5) as mentioned above (see Table I). In this table, the number 5 is allocated against the bicycle system which is absolutely environment-friendly and directly related to solar capital and the other end is allocated to the metro system which is normally driven by high-quality energy. The degree of Sustainability for different transportations systems are as in Fig. 1.

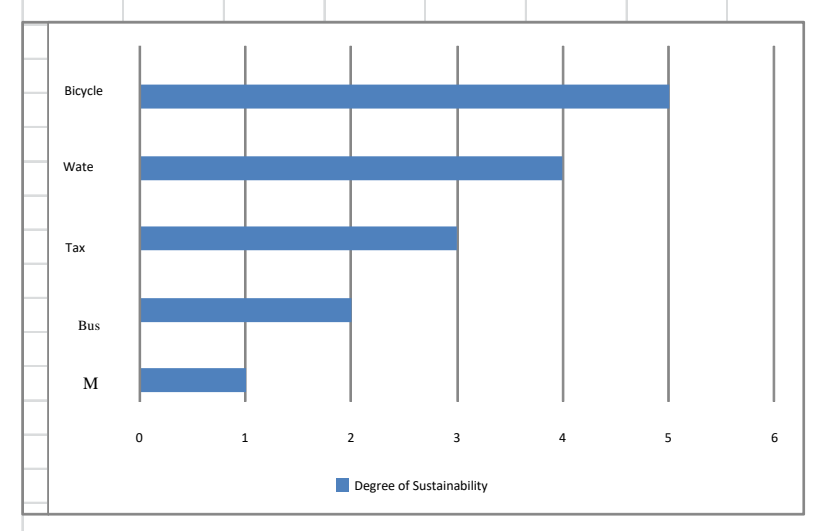

Fig. 1. The proposed degrees of sustainability for different transportation systems.

\section{The StUdy APPROACH}

Two oil-rich Iraqi governorates and the capital city Baghdad have been considered for simulation in this model, they are Maysan in the south and Erbil in the north of Iraq, both have wide roads, all are hard stricken war cities, followed by constant cut off for electrical supply for domestic and industrial usages and of course the ever hard economic situation for both local administrations and populations alike. On the other hand, Dubai the dynamic gulf state city was taken as a live example with population exceeding the one million and more with high Gross National Product GNP and continued economic growth and development, and of course of high carbon footprint in the whole gulf region.

Another good example for European cities, Glasgow was considered for simulation for the deduced model, it is an old European city, where limited and narrow roads and experiencing a postindustrial era was also a good example for consideration.

The ever increasing population, the immigration from countryside to cities, the usage of oil to boost development and the resulting environmental pollution and the increasing demand for energy, the degradation of environmental systems due to the irresponsible unstable and crippled economic growth and lack of corporate social responsibilities in most countries of the world particularly Iraq, all led to pursue this study,

The study dealt with Amara Maysan of Iraq southern city and Erbil for Iraq Kurdistan city north of Iraq, the two models for the pursued study at Iraq. Due to the vast regional and social differences between the two regions of UK in Europe, and the other selected cities in the Middle East. It is a trade-off, where environmental management is strongly implemented in one side and the war-stricken lawless Iraq is on the other side. This study discusses the different cases shadowed by the state of the fact findings. Based on the findings some suggested conclusive remarks were drawn accordingly as guidelines to adopt towards sustainable traffic systems. The side effect of any transportation system has been modeled where (train or Metro $=1$, Bus $=2$, Taxi=3, water transport=4, and Bike=5) [2], in that study the environmental impact of each transportation system (BSI) has been represented by (2).

\section{SUSTAINABILITY TRANSPORTATION AND TRAFFIC:}

In this model, 1 to 5 scale is being considered, this scale is introduced based on the degree of high quality and low quality energy used in driving the transportation system. Where 5 is classified as of sustainable impact, because of the fact low quality energy is utilized (the solar capital), in the other hand, metro system reflects the very bad impact that is because of using very high quality energy consumption, in this system oil energy is passed through various processes to provide electricity to drive the metro system; thus more energy consumption which leads to bad effect on the environment.

Reference [2] presented the BSI the Bicycle Sustainable Impact. The BSI was proposed to be directly proportional to the carrying capacity $\mathrm{n}$ and its degree of sustainability or impact on the environment I, the constant of proportionality is put equal to (1/5). Thus BSI is given by (2):

$$
B S I=\frac{n I}{5}
$$


where $n=$ the number of individuals carried by the transportation system, in this case either of ( 1 for bike, 20 for water boat, 4 for taxi, 50 for bus and 6 wagonX50 passengers for Metro system), and I is the degree of environmental sustainability or impact scale (1 to 5) (see Table I).

It is therefore, Table II can be developed using (2) and Table I to estimate the BSI for the five transportations systems.

TABLE II: SHOWS THE CALCULATED BSI FOR DIFFERENT TYPES OF TRANSPORTATION SYSTEMS

\begin{tabular}{cc}
\multicolumn{2}{c}{ TRANSPORTATION SYSTEMS } \\
\hline \hline Type of Transportation & BSI \\
\hline Bicycle & 1 \\
Water boat & 16 \\
Taxi car & 2.4 \\
Bus & 20 \\
Metro 6 wagons & 60 \\
\hline \hline
\end{tabular}

In order to highlight the negative side of sustainability, the Anti-Bike Sustainable Impact ASI is introduced in (3).

The Anti-Bike Sustainable Impact ASI (Anti-Bicycle Sustainable Impact) is then given by:

$$
A S I=\frac{n I}{5}-B S I_{\text {bicycle }}
$$

But $B S I_{\text {bicycle }}=1$, since $\mathrm{n}=1$ and $\mathrm{I}=5$, Thus Anti-Bike Sustainable Impact ASI is given by:

$$
A S I=\frac{n I}{5}-1
$$

In order to apply such model to the five selected cities, the above matrix (see Table III) is specified for the usages of different types of transportation systems for various selected cities

To compare the Anti-sustainability effect for the selected transportation system, equation (4) and Table III have been used to calculate the resulting Anti-Sustainability Impact ASI in accordance with the modes of the transportation systems that are being used in the different cities. The sum of individual substitution for each mode of transportation in (4) for each city was evaluated and graphed (see Fig. 2).

TABLE III: SHOWS DIFFERENT TYPES OF TRANSPORTATION BEING USED IN DIFFERENT CITIES

\begin{tabular}{lccccc}
\hline \hline CITY & BIKE & WATER & TAX & BUS & METRO \\
Erbil & 0 & 0 & 1 & 1 & 0 \\
Baghdad & 0 & 1 & 1 & 1 & 0 \\
Maysan & 0 & 0 & 1 & 0 & 0 \\
Dubai & 0 & 1 & 1 & 1 & 1 \\
Glasgow & 1 & 1 & 1 & 1 & 1 \\
\hline \hline
\end{tabular}

The ASI is evaluated and plotted for the various five cities of different modes of transportations using Table III and (4), for further clarifications, As an example, Baghdad city deploying Water, Taxi and Bus transportation systems, values for ( $\mathrm{n}, \mathrm{I})$ taken from Table III as $(20,4 \quad 4,3 \quad 50,2)$ substituted in (4), ASI = 35.4.

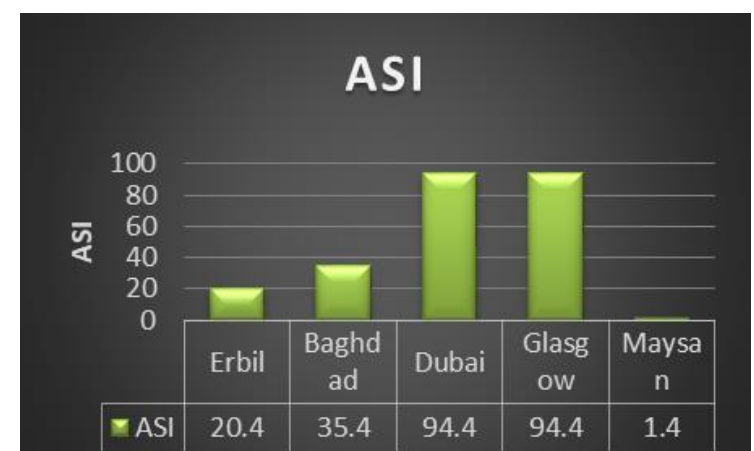

Fig. 2. The anti-sustainable impact due to different modes of transportation and for various cities.

The percentage of ASI (Unsustainability) indicator or an index (ASI-index) for the Anti-Sustainability can be evaluated by dividing the ASI for each transportation system by the ASI max $_{\text {of }}$ of the metro system (5):

$$
\text { ASI-index }=\%(\text { Anti }- \text { sust. })=\frac{A S I}{A S I_{\operatorname{Max}}}
$$

The percentage of Anti-Sustainability Impact for the different five types of transportation systems (see Fig. 3).

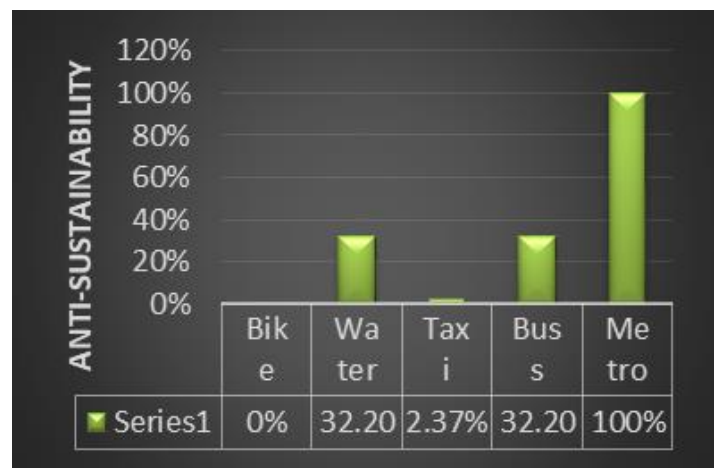

Fig. 3. The percentage of anti-sustainability (ASI-index ) for different transportation systems.

The term Anti-Sustainability is introduced to guide decision makers to reduce this peak rise in ecological footprint of some cities. It can be clearly said (see [. 2), that both Dubai and Glasgow constitute higher Anti-Sustainable Impact; this has been justified by the usage of metro system that leads to higher use of high quality energy, while Amara Maysan was the most distinguished city for the low ASI of 1.4; that is due to usage of taxi transportation only. The developed model represented by (4) and (5) can be implemented to any city using different transportation system. For future research, the Anti-Sustainable Impact ASI can be developed further by introducing non-linearity to the parameter $n$. this parameter will include multiple of decaying function that will take into account many modifications to the energy input, system efficiency and renewable energy usage.

\section{CONCLUSION}

The following can be concluded out of this paper:

A linear mathematical model has been constructed to 
mitigate the anti-sustainable impact for different cities of various transportation systems.

Different cities using different types of transportation system have been characteristically simulated as the most environment non-friendly cities.

The Anti-Sustainable Impact or unsustainability is being characterized by the usage of multiple transportation systems for the more developed cities of Glasgow and Dubai.

The high percentage of Anti-Sustainability is due to the metro deployment transportation system.

Using the derived model, it is possible to be implemented to estimate the Anti-Sustainability due to different modes of transportation deployed in any city.

Further future development for this basic linear model can be done. This development should include further elaboration on the introduction of non-linearity to the parameter $\mathrm{n}$.

\section{ACKNOWLEDGMENT}

The author would like to thank the Interior Design Department/ Faculty of Engineering/ ISHIK University for the use of the services and place to pursue this work.

My thanks also due to Mr. Behcet Celik,/Comp. Dept. for revising the text and Ms. Farah Maysan (Research Assistant) Interior Design Dept. / ISHIK University for the valuable support throughout the writing process.

\section{REFERENCES}

[1] H. M. Hassoon, "Lectures notes for environmental engineering course," Civil Engineering Dept., ISHIK University, Erbil, Iraq, Spring 2016.

[1] H. H. ALDelfi, "Linear modeling for sustainable transportation system," Eurasian Journal of Science \& Engineering, ISSN 2414-5629 (Print), ISSN 2414-5602 (Online), 2017.

[2] T. Y. Ceiang and M. J. Hsiao, "Carry-select adder using single ripple carry adder," Electron. Lett., vol. 34, no. 22, pp. 2101-2103, Oct. 1998.

[3] G. T. Miller and S. E. Spoolman, Living in the Environment, 17th ed, London, U.K: Brooks/Cole, Cengage Learning, pp. 5-9

[4] G. T. Miller and S. E. Spoolman, Living in the Environment, ch. 1, pp. 17.
[5] Y. Yevdokimov and H. Mao. (2004). Modeling sustainable transportation systems. [Online]. Available: http://www.unb.ca/research/transportation-group/_resources/pdf/resea rch-papers/modelingsustainable-transportation-systems.pdf

[6] R. Gilbert, N. Irwin, and B. Hollingsworth. (2002). Sustainable transportation performance indicators (STPI). [Online]. Available: http://www.richardgilbert.ca/Files/2003/Sustainable\%20Transportatio n\%20Performance\%20Inidicators\%20 (for\%20TRB).pdf

[7] R. Gilbert, N. Irwin, B. Hollingsworth, and P. Blais. (2002) Sustainable Transportation Performance Indicators,(STPI). [Online]. Available:

http://citeseerx.ist.psu.edu/viewdoc/download?doi=10.1.1.661.7698\& rep=rep $1 \&$ type $=$ pdf

[8] Dow Jones INDEX. (1999). Dow Jones Sustainability Indices. [Online]. Available:

https://en.wikipedia.org/wiki/Dow_Jones_Sustainability_Indices

[9] Dow Jones INDEX. (1999). Dow Jones Sustainability Indices. [Online]. Available:

https://en.wikipedia.org/wiki/Industry_Classification_Benchmark

[10] G. T. Miller and S. E. Spoolman, Living in the Environment, 2018, ch. 1, pp. 6-8.

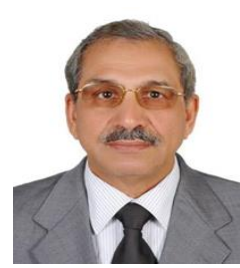

Hassan M. Hassoon ALDelfi was birn in Iraq, 1948. $\mathrm{He}$ got the $\mathrm{PhD}$. in mechanical engineering / ENV. Eng., Bristol University, Bristol, UK., 1989; the MSc in environmental engineering, Strathclyde University, Glasgow, UK, 1981; the BSc in mechanical engineering, London University, London, UK., 1972. $\mathrm{He}$ is an assoc. professor, in Interior Design Dept. Ishik University, Erbil, Iraq; regional manager of Ian Banham and Associates Consulting Engineering Dubai, Iraq Branch; director of Institutional Effectiveness, University of Dubai; head of higher studies, College of Mechanical Engineering, University of Technology; maintenances engineer, Daura Oil Refineries Baghdad Iraq; consulting design engineering in building services at Belyouha Consulting Architectural and Design Engineering, Deira, Dubai, UAE; design engineer, BOC-Linde Refrigeration limited Middlesex London UK; TRAINEE, British Steel Corporation North Wales UK.

Best research paper: Technology Research \& Paper by the $5^{\text {th }}$ annual international conference on architecture and civil engineering ACE 2017, Singapore. Dr. ALDelfi Awarded by the president of university of Dubai for completing ten years of work in the institution. He is awarded by Ishik university for contribution for obtaining the university international accreditation for his contribution as a team member and reporter. In addition to many awards in university committees like the International Relation Office IROW, the Interior Design Department as head of Scientific Committee. 